\title{
Uso de las WebQuest como recurso educativo Potencializador del aprendizaje De la Trigonometría ${ }^{1}$
}

\author{
Using WebQuest as an educational resource for \\ learning Potentiator From Trigonometry
}

Utilizando WebQuest como um recurso educacional para o aprendizado Potenciador De do trigonometria

Recibido: mayo de 2013

Aprobado: agosto de 2013
Sandra Marian Morales Munera ${ }^{2}$

Yeisson Alexis Acevedo Agudelo ${ }^{3}$

\section{Resumen}

El presente trabajo hace una descripción del análisis efectuado en estudio y aplicación de la metodología WebQuest como un recurso didáctico cuya fuente de información es Internet. El propósito de esta estrategia es elaborar un Situación didáctica usando el método WebQuest como estrategia de enseñanza enfocada en la investigación que centra su interés en el aprendizaje por descubrimiento guiado. Por lo cual se diseñó una situación didáctica de Trigonometría que busca desarrollar y fortalecer el trabajo autónomo, habilidades para la investigación y la toma de decisiones.

Palabras clave: Situación Didáctica; aula; recursos didácticos; recursos informáticos; TIC; aprendizaje por descubrimiento; WebQuest; matemáticas escolares; geometría; trigonometría.

\begin{abstract}
This paper is a description of the analysis in study and application of the methodology WebQuest as a teaching resource whose source of information is the Internet. The purpose of this strategy is to develop a teaching situation using the method WebQuest as a teaching strategy focused on research interest focuses on guided discovery learning. Therefore we designed a didactic situation Trigonometry that seeks to develop and strengthen self-employment, research skills and decision-making.

Keywords: Teaching Situation; classroom teaching resources, computing resources, ICT learning through discovery; WebQuest; school mathematics, geometry, trigonometry.
\end{abstract}

1 Artículo de Investigación

2 Universidad de Antioquia, Medellin. Colombia. Contacto: smoralesmunera@gmail.com

3 Universidad de Antioquia, Medellin. Colombia. Contacto: y.a.a.a@live.com 


\section{Resumo}

Este artigo é uma descrição da análise no estudo e aplicação da metodologia WebQuest como recurso didático cuja fonte de informação é a Internet. O objetivo desta estratégia é desenvolver uma situação de ensino utilizando o método WebQuest como estratégia de ensino focado no interesse de pesquisa se concentra no aprendizado descoberta guiada. Portanto, nós projetamos uma Trigonometria situação didática que busca desenvolver e fortalecer a auto-emprego, competências de investigação e de tomada de decisão.

Palavras-chave: situação de ensino, recursos didáticos em sala de aula, os recursos de computação, a aprendizagem das TIC através da descoberta; WebQuest; matemática escolar, geometria, trigonometria.

\section{Introducción}

En vista del creciente e inevitable impacto de las tecnologías de la información y la comunicación TIC, las escuelas deben integrar nuevos métodos de aprendizaje y enseñanza de tal forma que los estudiantes logren descubrir información y aprovechar todos los recursos disponibles en Internet, realidad que plantea Brunner,(2002) "la información es la materia prima para generar conocimiento" lo que implica una alfabetización tecnología, donde el estudiante interactúe con los diferentes tipos de herramienta y recursos los cuales permiten fortalecer la toma de decisión, el cuestionamiento, la autonomía entre otros.

La experiencia de aula se plantea debido a los deficientes resultados mostrados por los estudiantes de grado 10 de la Institución Educativa José Miguel de Restrepo y Puerta del Municipio de Copacabana durante el primer periodo de este año en el área de matemáticas, de un total de 170 estudiantes el 65\% aproximanente presenta deficiencia en dicha área.

Por lo tanto surge los siguientes interrogantes: ¿Cuáles pueden ser las posibilidades que ofrecen la TIC en la enseñanza de la trigonometría? ¿Qué beneficios aporta la estrategia por proyecto usando la metodología de trabajo WebQuest en la enseñanza de la trigonometría y como contribuye a la alfabetización de los estudiantes?, por lo tanto el propósito de esta estrategia es elaborar un Situación didáctica usando el método WebQuest como estrategia de enseñanza enfocada en la investigación que centra su interés en el aprendizaje por descubrimiento guiado.

\section{Marco Teóricos:}

Las WebQuest, Díaz,(2006) es la aplicación de una estrategia de aprendizaje por descubrimiento guiado a un proceso de trabajo desarrollado por los estudiantes utilizando los recursos de Internet . Originariamente fue formulado a mediados de los años noventa por Bernie Dodge (1995; 1998; 1999) de la Universidad de San Diego y desarrollado por Tom March $(1998 ; 2000)$ usando tareas dirigidas que poseen contenidos filtrados directamente por el docente, y lo que busca es guiar al estudiante en la búsqueda de Información confiable a través del uso de Internet, por tanto permite el manejo directo de la información y el desarrollo de habilidades con respecto a su manejo, generando en el estudiante la capacidad de análisis, síntesis y evaluación donde el mismo es consciente de su propio proceso de aprendizaje ya que el mismo focaliza la información en vez de buscarla.

Para Rodríguez García (s.f.) citado por Area (2004) se define la webquest asi:

- WebQuest es un modelo de aprendizaje extremamente simple y rico para propiciar el uso educativo de Internet, basado en el aprendizaje cooperativo y en procesos de investigación para aprender. 
- Un WebQuest es una actividad enfocada a la investigación, en la que la información usada por los alumnos es, en su mayor parte, descargada de Internet. Básicamente es una exploración dirigida, que culmina con la producción de una página Web, donde se publica el resultado de una investigación.

Por otro lado Adell (2004) resumen el concepto así "es una actividad didáctica que propone una tarea factible y atractiva para los estudiantes y un proceso para realizarla durante el cual, los alumnos harán cosas con información: analizar, sintetizar, comprender, transformar, crear, juzgar y valorar, crear nueva información, publicar, compartir, etc."

Además una WebQuest indaga a través de la creatividad y la innovación la forma de transmitir conocimientos adaptándose al contexto real y general de los estudiantes, permitiéndole al docente participar mediante la selección, el diseño, la secuencia y la aplicación del mismo para la generación del nuevo conocimiento para el alumno en trabajo colaborativo.

La estructura de una WebQuest adaptable a las necesidades debe partir de las necesidades e intereses de los estudiantes. Según José Sosa Díaz el esquema central sistematizado de la WebQuest yace inscrito en 6 componentes:

1) Introducción: Tópico de interés Información Básica, Motivación para el estudiante.

2) Tarea: Descripción clara y concisa sobre el producto a efectuar una vez finalizado el trayecto. Dogde (1995) nos ofrece este diagrama sobre las tareas que un alumno puede realizar en una WebQuest.

3) Proceso: Subtareas a efectuar, descomposición del tema siempre pensado hacia y desde la perspectiva de un aprendizaje cooperativo.

4) Recursos: Creación de enlaces a direcciones de internet Pensados para evitar que el alumno divague a la deriva a través de la red.
5) Evaluación: Establecimiento de criterios de evaluación precisos y específicos; uso de Rubricas. Las cuales según Popham,(1997), permiten que los maestros juzguen la idoneidad de las respuestas dadas por los estudiantes de una manera subjetiva.

6) Conclusión: Reflexión y resumen de la Experiencia, en esta el punto esencial es animar al alumno a seguir investigando.

\section{Conclusiones}

El procedimiento empleado en la realización de esta experiencia de aula constó de tres etapas: (a) la primera etapa estuvo dirigida al trabajo previo a la investigación, durante el cual se recolectó toda la información necesaria para la construcción de las WebQuests y la elaboración de las situaciones problema teniendo en cuenta los parámetros establecidos para la elaboración; (b) la segunda etapa consistió en la primera fase de la investigación propiamente dicha, durante ésta se validó la situación problema. (c) la última etapa que compone la segunda fase de la investigación la constituyó la aplicación de la situación problema a los cinco grupos del grado 10. Se considera la información proveniente a la observación directa, la observación participativa y los cuestionarios, se establecieron tres circunstancias relevantes para el análisis de los resultados: la primera corresponde a la validación de la situación problema, la segunda se refiere a la relación entre la situación problema en formato WebQuest y los estudiantes y la tercera a la relación que se produjo entre los estudiantes y el ambiente natural del aula de clase.

La WebQuest tiene una gran ventaja en su adaptabilidad a las necesidades de los estudiantes ya que permite la sistematización y ejecución de tareas determinadas y bien definidas, fomenta en el grupo estudiantil el trabajo colaborativo el cual potencializa pensamiento crítico.

A través de este recurso Educativo hemos encontrado que el estudiante se convierte en el protagonista absoluto de su proceso de aprendizaje. Se siente motivado por lo cual desarrolla su capacidad en la resolución de problemas a un mayor grado, 
mejorando sus destrezas para el análisis, síntesis y selección, ya que no busca respuestas por el contrario las fabrica.

\section{Referencias}

Adell, J. (2004): "Internet en el aula: las WebQuest", Edutec. Revista Electrónica de Tecnología Educativa, 17. Consultado en línea:http:// www.uib.es/depart/gte/edutec-e/revelec17/ adell_16a.htm. 15 de marzo de 2012.

Area Moreira, M. (2004a): WebQuest. Una estrategia de aprendizaje por descubrimiento, Quaderns Digitals, Monográfico WebQuest. Consultado: http://www.quadernsdigitals. net/index.php?accionMenu=hemeroteca. 30 de marzo de 2012.

Area Moreira, M. (2004b): Sociedad de la Información, Tecnologías digitales y educación: luces y sombras de una relación problemática. En García-Vera, B. Las nuevas tecnologías en la enseñanza (pp. 193-224).

Díaz Gutiérrez, E. J. (2006). El uso de WebQuest en la docencia universitaria: el aprendizaje colaborativo en red - Entorno WQ. Revista latinoamericana de Tecnología Educativa, 5 (2), 397-407.

Dodge, B. "Las WebQuest y el uso de la información" "Internet: oportunidades, límites y necesidad del Respeto", "Cinco reglas para escribir una fabulosa WebQuest". Consultar en: http:/www.eduteka.org. 1 de abril de 2012 .

Manrique, C. (2009). Sobre educación a distancia, conceptos, problemáticas y tendencias. Consultado en: http://blog.anced.org. pe/2009/01/20/sobre-educacion-a-distancia-conceptos-problematicas-y-tendencias/. 05 de Abril de 2012.

Popham, W.J. (1997). What's Wrong--and What's Right--with Rubrics. Educational Leadership, 55 (2). consultado en: http:// www.ascd.org/publications/educational-leadership/oct97/vol55/num02/ What's-Wrong\%E2\%80\%94and-What'sRight\%E2\%80\%94with-Rubrics.aspx. 11 de noviembre de 2011. 